\title{
Microvascular Endothelial Cells Exhibit Optimal Aspect Ratio for Minimizing Flow Resistance
}

\author{
Ronen Sumagin, ${ }^{1}$ Carl W. Brown III, ${ }^{1}$ Ingrid H. Sarelius, ${ }^{1,2}$ and Michael R. King ${ }^{1}$ \\ ${ }^{1}$ Department of Biomedical Engineering, University of Rochester, Rochester, NY 14627, USA; and ${ }^{2}$ Department of \\ Pharmacology \& Physiology, University of Rochester, Rochester, NY, USA
}

(Received 28 August 2007; accepted 7 February 2008; published online 4 March 2008)

\begin{abstract}
A recent analytical solution of the three-dimensional Stokes flow through a bumpy tube predicts that for a given bump area, there exists an optimal circumferential wavenumber which minimizes flow resistance. This study uses measurements of microvessel endothelial cell morphology to test whether this prediction holds in the microvasculature. Endothelial cell (EC) morphology was measured in blood perfused in situ microvessels in anesthetized mice using confocal intravital microscopy. EC borders were identified by immunofluorescently labeling the EC surface molecule ICAM-1 which is expressed on the surface but not in the EC border regions. Comparison of this theory with extensive in situ measurements of microvascular EC geometry in mouse cremaster muscle using intravital microscopy reveals that the spacing of EC nuclei in venules ranging from 27 to $106 \mu \mathrm{m}$ in diameter indeed lies quite close to this predicted optimal configuration. Interestingly, arteriolar ECs are configured to minimize flow resistance not in the resting state, but at the dilated vessel diameter. These results raise the question of whether less organized circulatory systems, such as that found in newly formed solid tumors or in the developing embryo, may deviate from the optimal bump spacing predicted to minimize flow resistance.
\end{abstract}

Keywords-Arteriole, Flow resistance, Intravital microscopy, Stokes flow, Venule.

\section{INTRODUCTION}

Within the circulation, the microvasculature is the primary site of regulation of many functions that are essential for homeostasis. For example, microvessels provide efficient transport to/from tissue of nutrients and metabolic products; exchange of respiratory gases; fluid volume regulation; and temperature regulation. As such, understanding the principles that underlie microcirculatory network organization and develop-

Address correspondence to Michael R. King, Department of Biomedical Engineering, University of Rochester, Rochester, NY 14627, USA. Electronic mail: mike_king@urmc.rochester.edu ment has been a target of ongoing investigation over many decades. A variety of explorations have defined important functional aspects of the fluid dynamics ${ }^{8,22}$ and gross morphology of the microcirculatory network. Thus key aspects of blood cell interactions ${ }^{7,22}$ and relationships between the overall network architecture and blood flow or energy minimization have been established, ${ }^{15,17}$ and a considerable amount is known about how local morphology at branches impacts the nature of the flow through them, both for large ${ }^{3,30}$ and small ${ }^{6,11}$ branches of the vascular tree. On a micro scale, consequences for the local shear field of small-scale irregularities in the surface of endothelial cells (ECs) have been explored. ${ }^{2}$ On the scale of individual microvessels, we ${ }^{29}$ and others ${ }^{1,10}$ have observed that microvessels have characteristic endothelial morphology, and, importantly, this morphology varies with position in the microvascular network. Whether this morphology has functional consequences is unknown.

A recent closed form solution of the three-dimensional Stokes flow through a bumpy tube ${ }^{25}$ allows an analysis of whether the local geometry of skeletal muscle arterioles and venules exists in an optimized state to maximize blood flow through individual microvessels for a given pressure drop. In this paper we describe extensive new measurements of EC dimensions within intact arterioles and venules in the cremaster muscle of anesthetized mice using intravital microscopy. We have compared this to the Stokes flow solution of Wang ${ }^{25}$ to determine how well the theory of bumpy tube flow predicts experimentally observed EC morphology with consequent characteristic distributions for the location of nuclei and hence "bumps" on the vessel wall. Indeed, we find that venular EC dimensions are very well predicted by the Stokes flow theory for a bumpy tube, and that arterial EC morphology is consistent with minimized flow resistance at maximally dilated, rather than resting diameter. 


\section{METHODS AND MATERIALS}

\section{Three-Dimensional Stokes Flow in a Bumpy Tube}

In a recent paper, Wang ${ }^{25}$ considered the threedimensional Stokes flow in a periodic bumpy-walled tube. Previous analytical investigations considered tubes with wall corrugations in one direction only, either in the longitudinal ${ }^{19,21}$ or transverse ${ }^{4,18}$ direction. The model of Wang consists of a tube wall with periodic bumps described by the non-dimensionalized equation

$$
r=1+\varepsilon \sin (n \theta) \sin (\alpha z)
$$

where the radial $(r)$ and longitudinal $(z)$ spatial variables have been scaled by the mean tube radius $a$, the bump amplitude is $\varepsilon=b / a \ll 1, n$ is the circumferential wavenumber, $\alpha=2 \pi a / l \neq 0$, and $l$ is the longitudinal wavelength. By performing a regular perturbation in the small parameter $\varepsilon$, Wang was able to obtain a closed form solution for the velocity distribution in terms of modified Bessel functions up to the second order correction to the mean flow rate. Interestingly, this solution predicts that for a given dimensionless bump area $A=\pi^{2} / n \alpha$, there exists an optimal circumferential wavenumber $n$ for which the flow resistance is minimized (or, equivalently, the flow rate maximized for a given total pressure drop). In this paper, we compare these predicted optimal bump size and spacings to experimental measurements made in intact mouse microvessels to determine how closely the Stokes flow solution correctly anticipates the vessel geometry, where the EC nuclei are taken to represent the quasi-periodic "bumps". Note that there are no adjustable parameters in comparing this theory to the experimental measurements.

\section{Animal Preparation}

All procedures were approved by the Institutional Review Board of the University of Rochester. C57BL6J mice (Jackson Laboratories) were initially anesthetized with sodium pentobarbital $(65 \mathrm{mg} / \mathrm{kg}$ ip) and maintained on supplemental anesthetic via a jugular catheter throughout the experiment. To insure a patent airway during the experiment, an endotracheal tube was inserted. Body temperature was maintained by placing the animal on a warmer during the experiment. The cremaster muscle was exposed using established methods. ${ }^{12,13}$ Briefly, the right cremaster muscle was exteriorized and laid flat on a quartz pedestal. During preparation and observation the tissue was continuously superfused with warmed physiological solution with the following composition: (in $\mathrm{mM}$ ) $\mathrm{NaCl}, 131.9$; $\mathrm{KCl}, 4.7 ; \mathrm{CaCl}, 2.0 ; \mathrm{MgSO}_{4}, 1.2, \mathrm{NaHCO}_{3}, 18 ; \mathrm{pH} 7.4$ at $36{ }^{\circ} \mathrm{C}$, and equilibrated with gas containing $0 \% \mathrm{O}_{2}$,
$5 \% \mathrm{CO}_{2}$, and $95 \% \mathrm{~N}_{2}$ to maintain tissue $\mathrm{PO}_{2}<15$ torr. Upon completion of the protocols, animals were euthanized by anesthetic overdose.

All animals received an intrascrotal injection of mouse recombinant $\mathrm{TNF} \alpha(0.5 \mu \mathrm{g} \mathrm{TNF} \alpha$ in $0.20 \mathrm{~mL}$ saline; Sigma-Aldrich) $3 \mathrm{~h}$ prior to the start of the surgical preparation to induce inflammatory conditions and to increase the expression of ICAM-1. ${ }^{12,23}$

\section{Immunofluorescence Labeling}

The approach to in vivo labeling and imaging of blood perfused microvessels has been described elsewhere ${ }^{12,23}$ : we have shown previously ${ }^{23}$ that as ICAM-1 is excluded from EC junctional regions, immunofluorescent labeling of ICAM-1 on the EC surface will serve to identify the morphology of individual ECs. Briefly, ICAM-1 expressed on the surface of the endothelium was labeled by localized perfusion using micropipette cannulation. Initially the vessel was perfused with rat anti-mouse monoclonal antibody in saline (YN/1.7.4, eBioscience, $50 \mu \mathrm{g} / \mathrm{mL}$ ) for $15 \mathrm{~min}$; the first pipette was withdrawn and blood perfusion briefly restored before the second cannulation with the pipette containing goat anti-rat secondary fluorescent polyclonal antibody in saline (Alexa 488 anti-rat, Molecular Probes, $50 \mu \mathrm{g} / \mathrm{mL}$ ), which was perfused for another $15 \mathrm{~min}$. Following this perfusion, the micropipette was withdrawn and blood flow was reestablished in the target microvascular region. During the cannulation the main arteriole was occluded by another micropipette upstream of the cannulation site at the time of the antibody loading to allow a complete perfusion of the downstream regions with the antibody solution. Intravascular pressure during perfusion with the antibody solutions is typically of the order of $20 \mathrm{~cm} \mathrm{H}_{2} \mathrm{O}$ in arterioles and $8 \mathrm{~cm} \mathrm{H}_{2} \mathrm{O}$ in venules ${ }^{20}$ which is in the physiological range. After antibody labeling and re-establishment of blood perfusion, microvessel diameters are typically not different from diameters before the labeling protocol.

\section{Intravital Microscopy and Image Analysis}

All images were acquired on an Olympus BX50WI microscope through an Olympus PlanF1 immersion objective ( $20 \times, 0.65 \mathrm{NA})$, giving a spatial resolution in our imaging system of $0.5 \mu \mathrm{m}$. Images were recorded to half inch SVHS (SONY VO9500) for offline analysis. Fluorescence images were collected from tissue that was illuminated with a $20 \mathrm{~mW}$ Argon laser and were acquired through a Nipkow disk scanning confocal head (CSU 10, Yokogawa) connected to an intensified CCD camera (XR Mega10, Stanford Photonics). Laser power and camera gain settings were held constant throughout the whole set of experiments. 
EC morphometry was measured using ImageJ software routines, and statistical analyses were completed using Prism (v4.0) software (Graphpad).

EC morphometry: To visualize ICAM-1 labeling, hence EC morphometry, images of intact blood perfused microvessels were captured and digitalized to 8 bit tiff images using a CG-7 frame grabber (Scion Corporation). Measurement of the EC dimensions was based on our previously published observation, where we showed that ICAM-1 labels the EC surface area while leaving the junctional areas unstained. ${ }^{23}$ In that study we also confirmed that excluded EC borders were indeed the junctional area by labeling the ECs for VE cadherin, a protein that is expressed exclusively in EC junctions, and confirming that both ICAM-1 and VE-cadherin labeling gave identical measurements of EC geometry. Briefly, to measure the area of ECs, the borders of individual ECs that were in clear focus were outlined using ImageJ software and the areas were calculated. Length and width of ECs were measured by projecting a line across the longest major and longest minor axis of the cells as demonstrated in Fig 1. In general, the major and minor cell axes corresponded to the flow and circumferential directions, respectively, to within $5^{\circ}$ in orientation. In the nomenclature of the

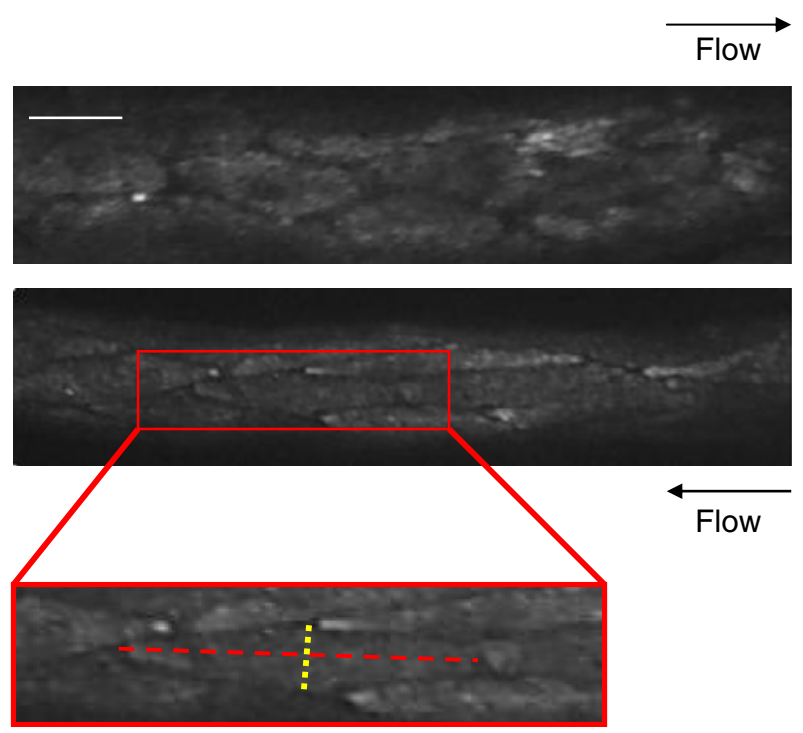

FIGURE 1. Exclusion of ICAM-1 staining from the EC borders can be used as a tool for determination of EC size and shape. Shown above are representative images of a venule (upper panel) and arteriole (lower panel) labeled for ICAM-1. Vessels were immunofluorescently labeled for ICAM-1 and imaged using intravital confocal microscopy as described in the METHODS AND MATERIALS section. Note the differences in shapes between the arteriolar and venular ECs. The bar in the upper panel is $20 \mu \mathrm{m}$ and represents both panels. EC length and width were measured by projecting a line across the longest major and minor EC axis as illustrated in the zoom-in image by red and yellow dotted lines respectively. Flow direction is indicated by the arrow.
Stokes flow theory, the major axis measured from individual EC dimensions becomes the $l$ parameter, while the theoretical circumferential wavenumber $n$ is determined by taking the individual vessel circumference (i.e., as calculated from the diameter) and dividing by the minor axis of the EC.

\section{Statistics}

Statistical tests were performed using Graphpad Prism (v4.0) to undertake $t$-tests and least squares linear regression of Fig. 2. Statistical significance was set at $p<0.05$.

\section{RESULTS AND DISCUSSION}

\section{EC Dimensions}

A major role of the microvascular network is to increase the total surface area available for exchange, which is essential for maintenance of proper tissue homeostasis. Since microvessel diameters range from a few micrometers in capillaries to hundreds of micrometers in the arterioles and venules, we asked the question of whether individual EC dimensions are preserved within this range of vessel sizes and whether this differs between arterioles and venules. To address this question, we labeled the microvessels for ICAM-1 (see METHODS) outlining the borders of the ECs, which allowed us to measure the length and width and to calculate the area of individual ECs. As illustrated in Fig. 1, EC shape is distinctively different in arterioles, which are characteristically elongated (major axis $79.0 \pm 1.4, n=200 ;$ minor axis $14.3 \pm 0.3 \mu \mathrm{m}$, $n=200$ ) compared to venules (major axis $47.3 \pm 1.2$, $n=110$; minor axis $23.1 \pm 0.5 \mu \mathrm{m}, n=110$ ). As shown in Fig. 2(a, d), the area of ECs did not remain constant throughout the microvasculature, but instead significantly increased with increasing vessel diameter in both arterioles $(p<0.0001$, correlation coefficient $\left.r^{2}=0.32\right)$ and venules $(p<0.001$, correlation coefficient $\left.r^{2}=0.13\right)$. Interestingly, the increase in EC area occurred differently in arterioles vs. venules. While arteriolar ECs are elongated, significantly increasing their major axis length across the range of diameters that we studied ( $p<0.0001, r^{2}=0.33$, Fig. $\left.2 b\right)$, venular ECs were wider and more round in larger vessels ( $p<0.0001, r^{2}=0.28$, Fig. 2f) leaving their major axis unchanged across all measured diameters.

While it is very likely that the differences in cell morphology in arterioles vs. venules is a direct result of characteristically different shear environment ${ }^{14}$ or the architecture of the vessel wall, it will most certainly create a different environment for leukocyte-EC interactions. For example it has been shown that in 

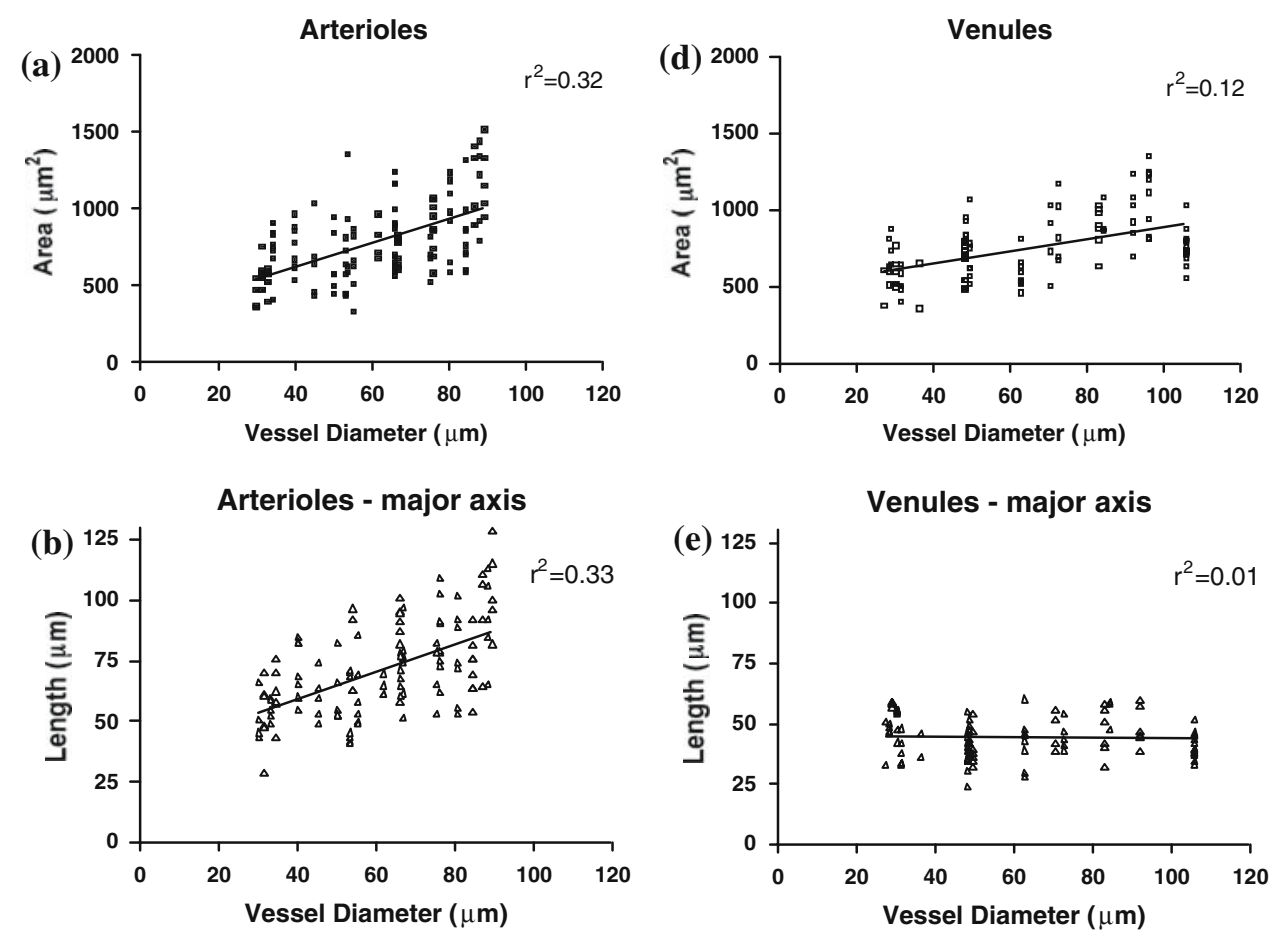

Arterioles - minor axis
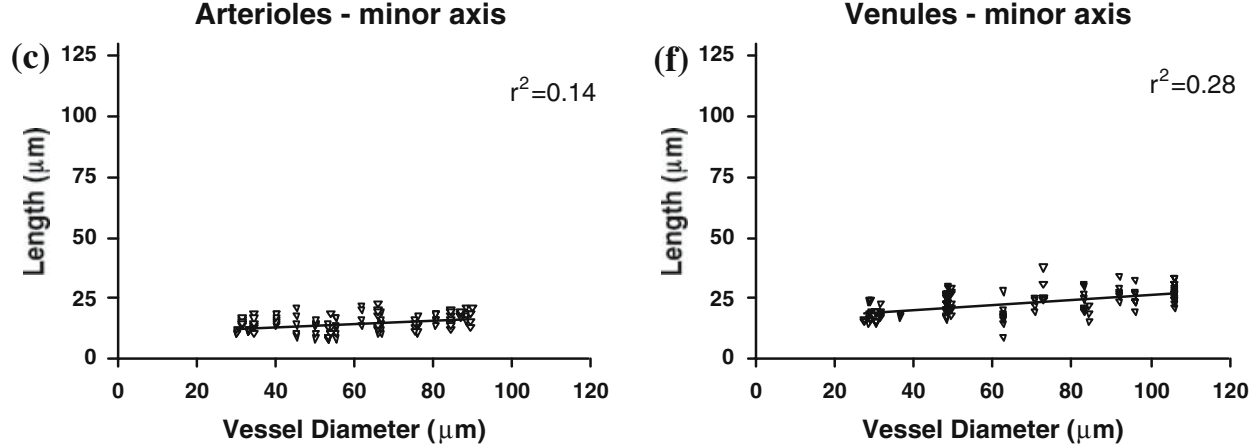

FIGURE 2. To measure the area of ECs in both arterioles (left panel) and venules (right panel), the microvasculature was labeled for ICAM-1 which is excluded from the junctional area, thus clearly outlining the cell borders. Individual cells were outlined using Image J software and cell area was calculated. Both arteriolar and venular ECs increased in size with an increase in vessel diameter. The increase in arterioles was much more pronounced. Importantly, with an increase in vessel size, arteriolar ECs elongated (b) while venular ECs became wider (f).

smaller venules where the junctional area per unit vessel length is significantly greater than in larger vessels, the probability that leukocytes will firmly adhere is significantly higher, resulting in a greater number of transmigrating leukocytes. ${ }^{29}$ Moreover, we have shown that expression levels and the distribution of adhesion molecules greatly affect the way leukocytes interact with the endothelium. ${ }^{23}$ For example in arterioles, where the expression of ICAM-1 is significantly lower than in venules following $\mathrm{TNF} \alpha$ treatment, leukocytes tend to roll but not to adhere as they do in venules. ${ }^{23}$ The EC morphology that we describe in the present study is likely an additional factor contributing to the differences in leukocyte behavior, and could be one of the reasons for the lack of leukocyte adhesion in activated arterioles. This is because the ratios of important adhesion molecules expressed on the surface of ECs (such as ICAM-1) vs. molecules expressed on EC junctional regions (such as VE-cadherin), and which are known to have signaling functions, ${ }^{27,28}$ will be different between narrow and long arteriolar ECs vs. wide and short venular ECs. We have shown previously that surface density of adhesion molecules directly correlate with leukocyte adhesion capability. ${ }^{24}$

Additionally, it has been demonstrated that when ICAM-1 is ligated by either leukocytes in vivo or antibodies in vitro ${ }^{26}$ it participates by mediating the signaling necessary for cytoskeletal changes which underlie the inflammatory response. Since ICAM-1 expression in arterioles is significantly lower than in 
venules ${ }^{23}$ elongated EC morphology that we described here allows longer interaction time between each leukocyte and EC enabling a higher degree of ICAM-1 engagement.

\section{Vessel Geometry is Optimized for Maintaining Proper Blood Flow}

When the optimal circumferential wavenumber (i.e., that which minimizes flow resistance) is plotted as a function of the dimensionless bump area $A$, a remarkable level of agreement is observed between the theoretically predicted optimal geometry and the experimentally measured microvascular EC dimensions (Fig. 3a). In Fig. 3, each symbol represents an individual EC measurement. Note that the venular EC
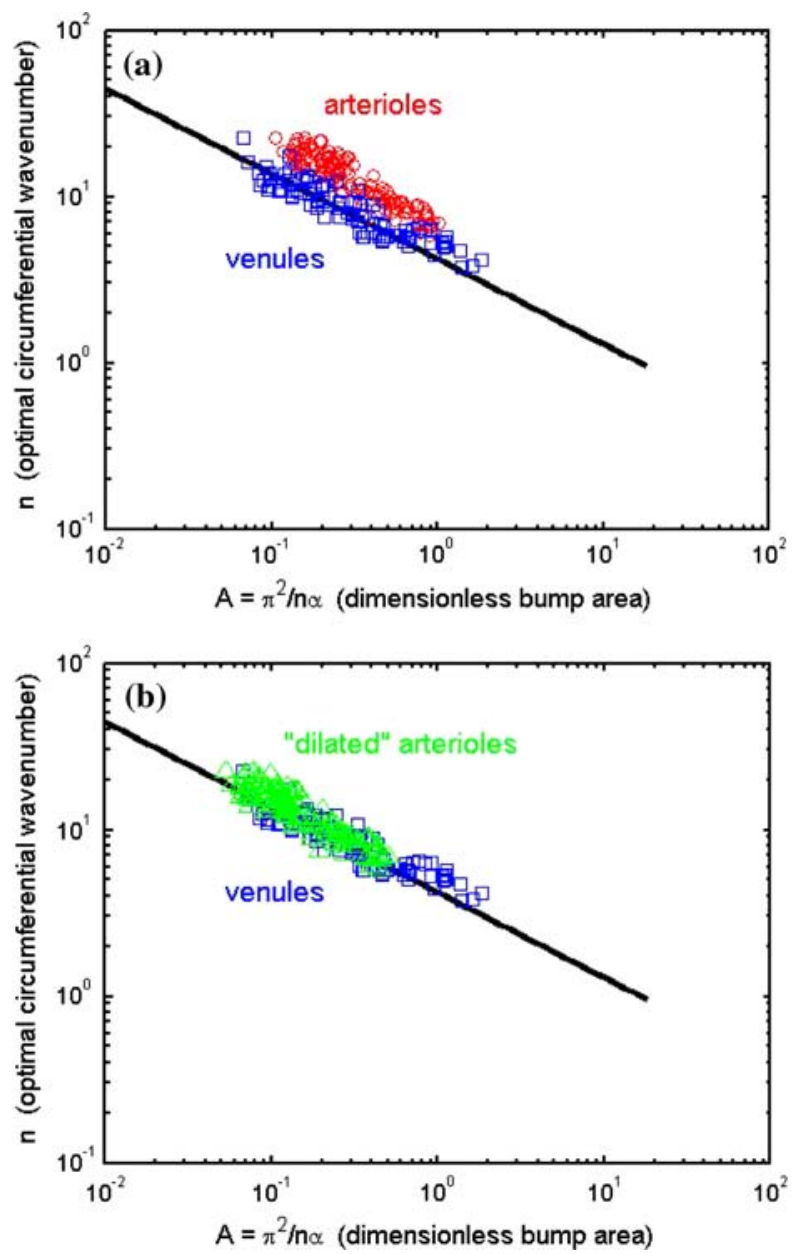

FIGURE 3. Comparison of the Stokes theory for threedimensional bumpy tube flow with experimentally measured microvessel geometries. (a) Blue squares represent venular EC dimensions while red circles represent arteriolar EC dimensions. (b) Arteriolar EC dimensions have been replotted (green circles) after increasing the vessel diameter by a factor of two to simulate physiological dilation. periodicity in the circumferential and axial directions is very well predicted from the Stokes flow theory, over a wide range of vessel diameters $(27-106 \mu \mathrm{m})$. The corresponding data measured from arterioles (red circles in Fig. 3a) lie somewhat above the theoretical curve, although follow the same trend over the observed range of arteriole diameters from 30 to $89 \mu \mathrm{m}$. Thus, at first it would appear that while venules exhibit EC dimensions very similar to the optimal bump geometry for minimization of flow resistance, arteriolar EC dimensions differ from the optimal geometry such that the circumferential wavenumber is somewhat higher than predicted for a given dimensionless bump area. However, it is well established that arterioles can dilate to as much as twice their resting diameter in response to metabolic or vasoactive stimuli. ${ }^{5,9,16}$ Thus, as the arteriolar dimensions measured in our present study are of vessels exhibiting resting tone rather than being actively dilated, we replotted the collected arteriolar data after scaling the vessel diameter by a factor of two, to approximate the corresponding arteriolar geometry after maximal vasodilation (Fig. 3b). Remarkably, this simple rescaling collapses the arteriolar data onto the predicted optimal configuration for minimization of flow resistance through a periodic bumpy tube. Thus, after accounting for the physiological regulation of microcirculatory blood flow via dilation and constriction of the arterioles, we may conclude from the present analysis that arterioles exhibit an EC geometry that is optimized for minimal flow resistance in the dilated, but not the resting state, which is perhaps expected since dilation and remodeling of arterioles towards their maximal diameter is usually associated with both acute responses (such as in exercise) or chronically (such as in hypertension or chronic inflammation) where rapid and efficient supply of oxygen or various inflammatory or stress-related factors is required. In contrast, venules (which do not exhibit active regulatory changes in diameter) exist in a single static geometry and are observed from this analysis to lie quite close to the optimal geometry predicted from Stokes flow theory.

\section{CONCLUSIONS}

In this paper, we have compared the predictions of the Stokes flow theory for three-dimensional bumpy tubes to novel measurements of microvascular EC dimensions in intact skeletal muscle of mouse. We find that these microvessels exhibit nearly optimal geometry for minimizing the overall flow resistance. These findings raise some intriguing questions which deserve further study. For instance, it would be interesting to characterize the microvascular EC geometry at 
different stages of embryonic development, to determine whether the microvasculature remodels from a non-optimized to optimized geometry over the weeks of gestation.

\section{ACKNOWLEDGEMENTS}

This work was supported by NIH Grant HL018208 and AHA Grant 0615677T.

\section{REFERENCES}

${ }^{1}$ Adamson, R. H. Microvascular endothelial cell shape and size in situ. Microvasc. Res. 46:77-88, 1993.

${ }^{2}$ Barbee, K. A., T. Mundel, R. Lal, and P. F. Davies Subcellular distribution of shear stress at the surface of flowaligned and nonaligned endothelial monolayers. Am. J. Physiol. 268:H1765-H1772, 1995.

${ }^{3}$ Caro, C. G., J. M. Fitz-Gerald, and R. C. Schroter Atheroma and arterial wall shear. Observation, correlation and proposal of a shear dependent mass transfer mechanism for atherogenesis. Proc. R. Soc. Lond. B Biol. Sci. 177:109$159,1971$.

${ }^{4}$ Deiber, J., and W. Schowalter Flow through tubes with sinusoidal axial variation in diameter. AIChE J. 25:638644, 1979.

${ }^{5}$ Duza, T., and I. H. Sarelius Increase in endothelial cell $\mathrm{Ca}(2+)$ in response to mouse cremaster muscle contraction. J. Physiol. 555:459-469, 2004.

${ }^{6}$ Frame, M. D., and I. H. Sarelius Arteriolar bifurcation angles vary with position and when flow is changed. Microvasc. Res. 46:190-205, 1993.

${ }^{7}$ Goldsmith, H. L., D. N. Bell, S. Spain, and F. A. McIntosh Effect of red blood cells and their aggregates on platelets and white cells in flowing blood. Biorheology 36:461-468, 1999.

${ }^{8}$ Goldsmith, H. L., G. R. Cokelet, and P. Gaehtgens Robin Fahraeus: evolution of his concepts in cardiovascular physiology. Am. J. Physiol. 257:H1005-H1015, 1989.

${ }^{9}$ Gorczynski, R. J., B. Klitzman, and B. R. Duling Interrelations between contracting striated muscle and precapillary microvessels. Am. J. Physiol. 235:H494-H504, 1978.

${ }^{10}$ Haas, T. L., and B. R. Duling Morphology favors an endothelial cell pathway for longitudinal conduction within arterioles. Microvasc. Res. 53:113-120, 1997.

${ }^{11}$ Kim, M. B., and I. H. Sarelius Distributions of wall shear stress in venular convergences of mouse cremaster muscle. Microcirculation 10:167-178, 2003.

${ }^{12} \mathrm{Kim}$, M. B., and I. H. Sarelius Role of shear forces and adhesion molecule distribution on P-selectin-mediated leukocyte rolling in postcapillary venules. Am. J. Physiol.: Heart Circ. Physiol. 287:H2705-H2711, 2004.
${ }^{13}$ Lau, K. S., R. W. Grange, E. Isotani, I. H. Sarelius, K. E. Kamm, P. L. Huang, and J. T. Stull nNOS and eNOS modulate cGMP formation and vascular response in contracting fast-twitch skeletal muscle. Physiol. Genomics 2:21-27, 2000.

${ }^{14}$ Lipowsky, H. H., S. Kovalcheck, and B. W. Zweifach The distribution of blood rheological parameters in the microvasculature of cat mesentery. Circ. Res. 43:738-749, 1978.

${ }^{15}$ Mayrovitz, H. N., and J. Roy Microvascular blood flow: evidence indicating a cubic dependence on arteriolar diameter. Am. J. Physiol. 245:H1031-H1038, 1983.

${ }^{16}$ Murrant, C. L., and I. H. Sarelius Local and remote arteriolar dilations initiated by skeletal muscle contraction. Am. J. Physiol.: Heart Circ. Physiol. 279:H2285-H2294, 2000.

${ }^{17}$ Murray, C. The physiological principle of minimum work applied to the angle of branching arteries. J. Gen. Phys. 9:835-841, 1926.

${ }^{18}$ Phan-Thien, N. On the Stokes flow of a viscous fluid through corrugated pipes. J. Appl. Mech. 47:961-963, 1980.

${ }^{19}$ Phan-Thien, N. On Stokes flows in channels and pipes with parallel stationary random surface roughness. Z. Ang. Math. Mech. 61:193-199, 1981.

${ }^{20}$ Sarelius, I. H., J. M. Kuebel, J. Wang, and V. H. Huxley Macromolecule permeability of in situ and excised rodent skeletal muscle arterioles and venules. Am. J. Physiol.: Heart Circ. Physiol. 290:H474-H480, 2006.

${ }^{21}$ Shaw, R., and A. London. Laminar Flow Forced Convection in Ducts. New York: Academic Press, 1978.

${ }^{22}$ Simon, S. I., and H. L. Goldsmith Leukocyte adhesion dynamics in shear flow. Ann. Biomed. Eng. 30:315-332, 2002.

${ }^{23}$ Sumagin, R., and I. H. Sarelius TNF-alpha activation of arterioles and venules alters distribution and levels of ICAM1 and affects leukocyte-endothelial cell interactions. Am. J. Physiol.: Heart Circ. Physiol. 291:H2116-H2125, 2006.

${ }^{24}$ Sumagin, R., and I. H. Sarelius A role for ICAM-1 in maintenance of leukocyte-endothelial cell rolling interactions in inflamed arterioles. Am. J. Physiol. Heart Circ. Physiol. 293:H2786-H2798, 2007.

${ }^{25}$ Wang, C. Y. Stokes flow through a tube with bumpy wall. Phys. Fluids 18:078101, 2006.

${ }^{26}$ Wang, Q., and C. M. Doerschuk Neutrophil-induced changes in the biomechanical properties of endothelial cells: roles of ICAM-1 and reactive oxygen species. $J$. Immunol. 164:6487-6494, 2000.

${ }^{27}$ Wang, Q., and C. M. Doerschuk The p38 mitogen-activated protein kinase mediates cytoskeletal remodeling in pulmonary microvascular endothelial cells upon intracellular adhesion molecule-1 ligation. J. Immunol. 166:68776884, 2001.

${ }^{28}$ Wojciak-Stothard, B., and A. J. Ridley Rho GTPases and the regulation of endothelial permeability. Vascul. Pharmacol. 39:187-199, 2002.

${ }^{29}$ Wojciechowski, J. C., and I. H. Sarelius Preferential binding of leukocytes to the endothelial junction region in venules in situ. Microcirculation 12:349-359, 2005.

${ }^{30}$ Zamir, M. Optimality principles in arterial branching. $J$. Theor. Biol. 62:227-251, 1976. 